\title{
Kinney Revisited: An Improved Group Contribution Method for the Prediction of Boiling Points
}

\section{of Acyclic Alkanes}

\author{
J. A. Palatinus, C. M. Sams, C. M. Beeston, F. A. Carroll, ${ }^{*}$ A. B. Argenton, and F. H. Quina
}

Table 1. Data and Parameters Used with Equations 3 and 4 and in Figure 1 for All Compounds in the Data Set.

\begin{tabular}{|c|c|c|c|c|c|c|c|c|c|c|c|c|c|}
\hline No. & Compound & $\mathrm{BP}(\mathrm{K})$ & $\underline{\text { Ref. }}$ & $\underline{C}$ & $\underline{H}$ & $\underline{M}$ & $\underline{E}$ & $\underline{P}$ & $\underline{D}$ & $\underline{V}_{2 \text { or } 3}$ & $\underline{\boldsymbol{V}}_{4+}$ & $\frac{\text { Kinney }}{\underline{Y}}$ & $\frac{\text { Pred. }}{\underline{\operatorname{BP}(K)}}$ \\
\hline 1 & hexane & 341.89 & w & 6 & 14 & 0 & 0 & 0 & 0 & 0 & 0 & 18.80 & 341.9 \\
\hline 2 & 3-methylpentane & 336.43 & W & 5 & 11 & 1 & 0 & 0 & 0 & 0 & 0 & 18.05 & 333.7 \\
\hline 3 & 2-methylpentane & 333.42 & W & 5 & 11 & 1 & 0 & 0 & 0 & 0 & 0 & 18.05 & 333.7 \\
\hline 4 & 2,3-dimethylbutane & 331.14 & W & 4 & 8 & 2 & 0 & 0 & 0 & 1 & 0 & 17.80 & 330.9 \\
\hline 5 & 2,2-dimethylbutane & 322.89 & W & 4 & 8 & 2 & 0 & 0 & 1 & 0 & 0 & 16.90 & 320.6 \\
\hline 6 & heptane & 371.58 & W & 7 & 16 & 0 & 0 & 0 & 0 & 0 & 0 & 21.60 & 370.9 \\
\hline 7 & 3-methylhexane & 365 & w & 6 & 13 & 1 & 0 & 0 & 0 & 0 & 0 & 20.85 & 363.4 \\
\hline 8 & 2-methylhexane & 363.2 & W & 6 & 13 & 1 & 0 & 0 & 0 & 0 & 0 & 20.85 & 363.4 \\
\hline 9 & 3-ethylpentane & 366.63 & W & 5 & 11 & 0 & 1 & 0 & 0 & 0 & 0 & 20.50 & 359.9 \\
\hline 10 & 2,3-dimethylpentane & 362.93 & W & 5 & 10 & 2 & 0 & 0 & 0 & 1 & 0 & 20.60 & 360.9 \\
\hline 11 & 3,3-dimethylpentane & 359.21 & W & 5 & 10 & 2 & 0 & 0 & 0 & 0 & 0 & 20.10 & 355.7 \\
\hline 12 & 2,4-dimethylpentane & 353.65 & W & 5 & 10 & 2 & 0 & 0 & 0 & 0 & 0 & 20.10 & 355.7 \\
\hline 13 & 2,2-dimethylpentane & 352.35 & W & 5 & 10 & 2 & 0 & 0 & 1 & 0 & 0 & 19.70 & 351.6 \\
\hline 14 & 2,2,3-trimethylbutane & 354.03 & W & 4 & 7 & 3 & 0 & 0 & 1 & 1 & 0 & 19.45 & 348.9 \\
\hline 15 & octane & 398.82 & W & 8 & 18 & 0 & 0 & 0 & 0 & 0 & 0 & 24.40 & 397.5 \\
\hline 16 & 3-methylheptane & 392.08 & W & 7 & 15 & 1 & 0 & 0 & 0 & 0 & 0 & 23.65 & 390.6 \\
\hline 17 & 4-methylheptane & 390.86 & w & 7 & 15 & 1 & 0 & 0 & 0 & 0 & 0 & 23.65 & 390.6 \\
\hline 18 & 2-methylheptane & 390.8 & W & 7 & 15 & 1 & 0 & 0 & 0 & 0 & 0 & 23.65 & 390.6 \\
\hline 19 & 3-ethylhexane & 391.68 & W & 6 & 13 & 0 & 1 & 0 & 0 & 0 & 0 & 23.30 & 387.3 \\
\hline 20 & 3,4-dimethylhexane & 390.38 & W & 6 & 12 & 2 & 0 & 0 & 0 & 1 & 0 & 23.40 & 388.3 \\
\hline 21 & 2,3-dimethylhexane & 388.76 & W & 6 & 12 & 2 & 0 & 0 & 0 & 1 & 0 & 23.40 & 388.3 \\
\hline 22 & 3,3-dimethylhexane & 385.12 & W & 6 & 12 & 2 & 0 & 0 & 0 & 0 & 0 & 22.90 & 383.5 \\
\hline
\end{tabular}


2,4-dimethylhexane

2,5-dimethylhexane

2,2-dimethylhexane

3-ethyl-3-methylpentane

3-ethyl-2-methylpentane

2,3,3-trimethylpentane

2,3,4-trimethylpentane

2,2,3-trimethylpentane

2,2,4-trimethylpentane

2,2,3,3-tetramethylbutane

nonane

3-methyloctane

2-methyloctane

4-methyloctane

3-ethylheptane

4-ethylheptane

3,4-dimethylheptane

2,3-dimethylheptane

3,3-dimethylheptane

2,5-dimethylheptane

3,5-dimethylheptane

2,6-dimethylheptane

4,4-dimethylheptane

2,4-dimethylheptane

2,2-dimethylheptane

3-ethyl-3-methylhexane

3,3,4-trimethylhexane

3-ethyl-4-methylhexane

2,3,4-trimethylhexane

3-ethyl-2-methylhexane

2,3,3-trimethylhexane

4-ethyl-2-methylhexane

2,2,3-trimethylhexane

2,3,5-trimethylhexane

2,4,4-trimethylhexane

2,2,4-trimethylhexane

2,2,5-trimethylhexane

3,3-diethylpentane

3-ethyl-2,3-dimethylpentane

2,3,3,4-tetramethylpentane

$\begin{array}{lll}382.58 & \text { W } & 6 \\ 382.25 & \text { W } & 6 \\ 381.99 & \text { W } & 6 \\ 391.41 & \text { W } & 5 \\ 388.8 & \text { W } & 5 \\ 387.91 & \text { W } & 5 \\ 386.62 & \text { W } & 5 \\ 382.99 & \text { W } & 5 \\ 372.39 & \text { W } & 5 \\ 379.62 & \text { W } & 4 \\ 423.95 & \text { W } & 9 \\ 417.36 & \text { W } & 8 \\ 416.41 & \text { W } & 8 \\ 415.57 & \text { W } & 8 \\ 416.15 & \text { W } & 7 \\ 414.35 & \text { W } & 7 \\ 413.75 & \text { W } & 7 \\ 413.65 & \text { W } & 7 \\ 410.16 & \text { W } & 7 \\ 409.15 & \text { W } & 7 \\ 409.15 & \text { W } & 7 \\ 408.36 & \text { W } & 7 \\ 408.35 & \text { W } & 7 \\ 406.04 & \text { W } & 7 \\ 405.84 & \text { W } & 7 \\ 413.75 & \text { W } & 6 \\ 413.61 & \text { W } & 6 \\ 413.55 & \text { W } & 6 \\ 412.19 & \text { W } & 6 \\ 411.15 & \text { W } & 6 \\ 410.83 & \text { W } & 6 \\ 406.95 & \text { W } & 6 \\ 406.75 & \text { W } & 6 \\ 404.49 & \text { W } & 6 \\ 403.8 & \text { W } & 6 \\ 399.69 & \text { W } & 6 \\ 397.23 & \text { W } & 6 \\ 419.32 & \text { W } & 5 \\ 417.85 & \text { W } & 5 \\ 414.7 & \text { W } & 5\end{array}$

$\begin{array}{ll}22.90 & 383.5 \\ 22.90 & 383.5 \\ 22.50 & 379.7 \\ 22.55 & 380.2 \\ 23.05 & 385.0 \\ 22.65 & 381.2 \\ 22.65 & 381.2 \\ 22.25 & 377.3 \\ 21.75 & 372.4 \\ 21.60 & 370.9 \\ 27.20 & 422.1 \\ 26.45 & 415.7 \\ 26.45 & 415.7 \\ 26.45 & 415.7 \\ 26.10 & 412.7 \\ 26.10 & 412.7 \\ 25.70 & 409.2 \\ 25.70 & 409.2 \\ 25.70 & 409.2 \\ 25.70 & 409.2 \\ 25.70 & 409.2 \\ 25.70 & 409.2 \\ 25.70 & 409.2 \\ 25.70 & 409.2 \\ 25.30 & 405.6 \\ 25.35 & 406.1 \\ 25.45 & 406.9 \\ 25.85 & 410.5 \\ 25.45 & 406.9 \\ 25.85 & 410.5 \\ 25.45 & 406.9 \\ 25.35 & 406.1 \\ 25.05 & 403.4 \\ 25.45 & 406.9 \\ 24.95 & 402.5 \\ 24.55 & 398.9 \\ 24.55 & 398.9 \\ 25.00 & 402.9 \\ 25.10 & 403.8 \\ 25.20 & 404.7\end{array}$


2,2,3,3-tetramethylpentane

3-ethyl-2,4-dimethylpentane

3-ethyl-2,2-dimethylpentane

2,2,3,4-tetramethylpentane

2,2,4,4-tetramethylpentane

decane

3-methylnonane

2-methylnonane

4-methylnonane

5-methylnonane

3-ethyloctane

2,3-dimethyloctane

4-ethyloctane

3,4-dimethyloctane

4,5-dimethyloctane

3,3-dimethyloctane

3,6-dimethyloctane

2,7-dimethyloctane

3,5-dimethyloctane

2,6-dimethyloctane

2,5-dimethyloctane

4,4-dimethyloctane

2,2-dimethyloctane

2,4-dimethyloctane

3-ethyl-3-methylheptane

3-ethyl-4-methylheptane

3,4,5-trimethylheptane

3,3,4-trimethylheptane

3-ethyl-2-methylheptane

3,4,4-trimethylheptane

4-ethyl-4-methylheptane

2,3,5-trimethylheptane

2,3,3-trimethylheptane

2,3,4-trimethylheptane

5-ethyl-2-methylheptane

3-ethyl-5-methylheptane

2,2,3-trimethylheptane

4-propylheptane

2,4,5-trimethylheptane

4-ethyl-2-methylheptane

413.42
409.84
406.98
406.17
395.43
447.27
440.95
440.15
438.85
438.25
439.65
437.46
436.79
436.55
435.28
434.35
433.95
433.02
432.55
431.69
431.65
430.65
430.05
429.05
436.95
436.15
435.65
435.05
434.35
434.25
433.95
433.85
433.35
433.05
432.85
431.35
430.75
430.65
429.65

24.80

25.10

24.70

24.80

23.40

30.00

29.25

29.25

29.25

29.25

28.90

28.50

28.90

28.50

28.50

28.50

28.50

28.50

28.50

28.50

28.50

28.50

28.10

28.50

28.15

28.15

27.75

27.75

28.15

27.75

28.15

27.75

28.25

27.75

28.15

28.15

27.35

27.60

27.75

28.15
401.1

403.8

400.2

401.1

388.3

445.1

439.1

439.1

439.1

439.1

436.2

433.0

436.2

433.0

433.0

433.0

433.0

433.0

433.0

433.0

433.0

433.0

429.7

433.0

430.1

430.1

426.8

426.8

430.1

426.8

430.1

426.8

430.9

426.8

430.1

430.1

423.4

425.5

426.8

430.1 


\begin{tabular}{|c|c|c|c|c|c|c|c|c|c|c|c|c|c|}
\hline 103 & 2,3,6-trimethylheptane & 429.15 & W & 7 & 13 & 3 & 0 & 0 & 0 & 0 & 0 & 27.75 & 426.8 \\
\hline 104 & 3,3,5-trimethylheptane & 428.83 & W & 7 & 13 & 3 & 0 & 0 & 0 & 0 & 0 & 27.75 & 426.8 \\
\hline 105 & 2,5,5-trimethylheptane & 425.95 & W & 7 & 13 & 3 & 0 & 0 & 0 & 0 & 0 & 27.75 & 426.8 \\
\hline 106 & 2,4,4-trimethylheptane & 424.15 & W & 7 & 13 & 3 & 0 & 0 & 0 & 0 & 0 & 27.75 & 426.8 \\
\hline 107 & 2,2,5-trimethylheptane & 423.95 & W & 7 & 13 & 3 & 0 & 0 & 1 & 0 & 0 & 27.35 & 423.4 \\
\hline 108 & 2,2,6-trimethylheptane & 422.08 & W & 7 & 13 & 3 & 0 & 0 & 1 & 0 & 0 & 27.35 & 423.4 \\
\hline 109 & 2,2,4-trimethylheptane & 421.45 & W & 7 & 13 & 3 & 0 & 0 & 1 & 0 & 0 & 27.35 & 423.4 \\
\hline 110 & 2,4,6-trimethylheptane & 420.75 & W & 7 & 13 & 3 & 0 & 0 & 0 & 0 & 0 & 27.75 & 426.8 \\
\hline 111 & 3,3,4,4-tetramethylhexane & 443.15 & W & 6 & 10 & 4 & 0 & 0 & 0 & 0 & 1 & 28.00 & 428.8 \\
\hline 112 & 3,3-diethylhexane & 439.45 & W & 6 & 12 & 0 & 2 & 0 & 0 & 0 & 0 & 27.80 & 427.2 \\
\hline 113 & 2,3,3,4-tetramethylhexane & 437.74 & W & 6 & 10 & 4 & 0 & 0 & 0 & 0 & 1 & 28.00 & 428.8 \\
\hline 114 & 3,4-diethylhexane & 437.05 & W & 6 & 12 & 0 & 2 & 0 & 0 & 1 & 0 & 28.30 & 431.3 \\
\hline 115 & 3-ethyl-2,3-dimethylhexane & 436.85 & W & 6 & 11 & 2 & 1 & 0 & 0 & 1 & 0 & 27.90 & 428.0 \\
\hline 116 & 4-ethyl-3,3-dimethylhexane & 436.05 & W & 6 & 11 & 2 & 1 & 0 & 0 & 1 & 0 & 27.90 & 428.0 \\
\hline 117 & 3-ethyl-3,4-dimethylhexane & 435.25 & W & 6 & 11 & 2 & 1 & 0 & 0 & 1 & 0 & 27.90 & 428.0 \\
\hline 118 & 2,3,4,4-tetramethylhexane & 434.75 & W & 6 & 10 & 4 & 0 & 0 & 0 & 0 & 1 & 28.00 & 428.8 \\
\hline 119 & 4-ethyl-2,4-dimethylhexane & 434.25 & W & 6 & 11 & 2 & 1 & 0 & 0 & 0 & 0 & 27.40 & 423.8 \\
\hline 120 & 4-ethyl-2,3-dimethylhexane & 434.05 & W & 6 & 11 & 2 & 1 & 0 & 0 & 1 & 0 & 27.90 & 428.0 \\
\hline 121 & 2,2,3,3-tetramethylhexane & 433.46 & W & 6 & 10 & 4 & 0 & 0 & 1 & 0 & 1 & 27.60 & 425.5 \\
\hline 122 & 3-ethyl-2,4-dimethylhexane & 433.25 & W & 6 & 11 & 2 & 1 & 0 & 0 & 1 & 0 & 27.90 & 428.0 \\
\hline 123 & 2,2,3,4-tetramethylhexane & 431.95 & W & 6 & 10 & 4 & 0 & 0 & 1 & 0 & 1 & 27.60 & 425.5 \\
\hline 124 & 2,3,4,5-tetramethylhexane & 429.35 & W & 6 & 10 & 4 & 0 & 0 & 0 & 0 & 1 & 28.00 & 428.8 \\
\hline 125 & 3-ethyl-2,2-dimethylhexane & 429.25 & w & 6 & 11 & 2 & 1 & 0 & 1 & 1 & 0 & 27.50 & 424.7 \\
\hline 126 & 3-ethyl-2,5-dimethylhexane & 427.25 & W & 6 & 11 & 2 & 1 & 0 & 0 & 1 & 0 & 27.90 & 428.0 \\
\hline 127 & 2,2,4,4-tetramethylhexane & 426.95 & W & 6 & 10 & 4 & 0 & 0 & 1 & 0 & 0 & 26.60 & 417.0 \\
\hline 128 & 2,3,3,5-tetramethylhexane & 426.25 & W & 6 & 10 & 4 & 0 & 0 & 0 & 1 & 0 & 27.50 & 424.7 \\
\hline 129 & 2,2,3,5-tetramethylhexane & 421.55 & W & 6 & 10 & 4 & 0 & 0 & 1 & 1 & 0 & 27.10 & 421.3 \\
\hline 130 & 2,2,4,5-tetramethylhexane & 421.03 & W & 6 & 10 & 4 & 0 & 0 & 1 & 1 & 0 & 27.10 & 421.3 \\
\hline 131 & 4-ethyl-2,2-dimethylhexane & 420.15 & W & 6 & 11 & 2 & 1 & 0 & 1 & 0 & 0 & 27.00 & 420.4 \\
\hline 132 & 2,2,5,5-tetramethylhexane & 410.61 & W & 6 & 10 & 4 & 0 & 0 & 2 & 0 & 0 & 26.20 & 413.5 \\
\hline 133 & 3,3-diethyl-2-methylpentane & 442.85 & W & 5 & 9 & 1 & 2 & 0 & 0 & 1 & 0 & 27.55 & 425.1 \\
\hline 134 & 3-ethyl-2,2,3-trimethylpentane & 442.65 & W & 5 & 8 & 3 & 1 & 0 & 1 & 0 & 1 & 27.25 & 422.5 \\
\hline 135 & 3-ethyl-2,3,4-trimethylpentane & 442.59 & W & 5 & 8 & 3 & 1 & 0 & 0 & 0 & 1 & 27.65 & 425.9 \\
\hline 136 & 2,2,3,3,4-pentamethylpentane & 439.2 & W & 5 & 7 & 5 & 0 & 0 & 1 & 0 & 1 & 26.85 & 419.1 \\
\hline 137 & 2,2,3,4,4-pentamethylpentane & 432.44 & W & 5 & 7 & 5 & 0 & 0 & 2 & 0 & 1 & 26.45 & 415.7 \\
\hline 138 & 3-ethyl-2,2,4-trimethylpentane & 428.45 & W & 5 & 8 & 3 & 1 & 0 & 1 & 0 & 1 & 27.25 & 422.5 \\
\hline 139 & undecane & 469.08 & W & 11 & 24 & 0 & 0 & 0 & 0 & 0 & 0 & 32.80 & 466.7 \\
\hline 140 & 3-methyldecane & 463.99 & $\mathrm{C}$ & 10 & 21 & 1 & 0 & 0 & 0 & 0 & 0 & 32.05 & 461.0 \\
\hline 141 & 2-methyldecane & 462.39 & $\mathrm{C}$ & 10 & 21 & 1 & 0 & 0 & 0 & 0 & 0 & 32.05 & 461.0 \\
\hline 142 & 2,3-dimethylnonane & 459.99 & C & 9 & 18 & 2 & 0 & 0 & 0 & 0 & 0 & 31.30 & 455.3 \\
\hline
\end{tabular}




$\begin{array}{ll}143 & \text { 2,2-dimethylnonane } \\ 144 & \text { dodecane } \\ 145 & \text { 3-methylundecane } \\ 146 & \text { 2-methylundecane } \\ 147 & \text { 2,3-dimethyldecane } \\ 148 & \text { 2,2-dimethyldecane } \\ 149 & \text { tridecane } \\ 150 & \text { 3-methyldodecane } \\ 151 & \text { 2-methyldodecane } \\ 152 & \text { 2,3-dimethylundecane } \\ 153 & \text { tetradecane } \\ 154 & \text { 3-methyltridecane } \\ 155 & \text { 2-methyltridecane } \\ 156 & \text { 2,3-dimethyldodecane } \\ 157 & \text { 4-propylundecane } \\ 158 & \text { 4,5-dipropyloctane } \\ 159 & \text { pentadecane } \\ 160 & \text { 3-methyltetradecane } \\ 161 & \text { 2-methyltetradecane } \\ 162 & \text { 2,3-dimethyltridecane } \\ 163 & \text { 6-propyldodecane } \\ 164 & \text { 4-methyl-6-propylundecane } \\ 165 & \text { hexadecane } \\ 166 & \text { 3-methylpentadecane } \\ 167 & \text { 2-methylpentadecane } \\ 168 & \text { 2,3-dimethyltetradecane } \\ 169 & \text { heptadecane } \\ 170 & \text { 2-methylhexadecane } \\ 171 & \text { 3-methylhexadecane } \\ 172 & \text { 2,3-dimethylpentadecane } \\ 173 & \text { octadecane } \\ 174 & \text { 3-methylheptadecane } \\ 175 & \text { 2-methylheptadecane } \\ 176 & \text { 2,3-dimethylhexadecane } \\ 177 & \text { nonadecane } \\ 178 & \text { 3-methyloctadecane } \\ 179 & \text { 2-methyloctadecane } \\ 180 & \text { 2,3-dimethylheptadecane } \\ 181 & \text { eicosane } \\ 182 & \text { 3-methylnonadecane } \\ & \end{array}$

\begin{tabular}{|c|c|c|c|c|c|c|c|c|c|c|c|}
\hline 450.19 & $C$ & 9 & 18 & 2 & 0 & 0 & 1 & 0 & 0 & 30.90 & 452.2 \\
\hline 489.47 & W & 12 & 26 & 0 & 0 & 0 & 0 & 0 & 0 & 35.60 & 487.1 \\
\hline 484.39 & C & 11 & 23 & 1 & 0 & 0 & 0 & 0 & 0 & 34.85 & 481.7 \\
\hline 483.39 & C & 11 & 23 & 1 & 0 & 0 & 0 & 0 & 0 & 34.85 & 481.7 \\
\hline 479.99 & C & 10 & 20 & 2 & 0 & 0 & 0 & 0 & 0 & 34.10 & 476.3 \\
\hline 472.19 & $C$ & 10 & 20 & 2 & 0 & 0 & 1 & 0 & 0 & 33.70 & 473.4 \\
\hline 508.62 & W & 13 & 28 & 0 & 0 & 0 & 0 & 0 & 0 & 38.40 & 506.4 \\
\hline 503.39 & $C$ & 12 & 25 & 1 & 0 & 0 & 0 & 0 & 0 & 37.65 & 501.3 \\
\hline 502.59 & $C$ & 12 & 25 & 1 & 0 & 0 & 0 & 0 & 0 & 37.65 & 501.3 \\
\hline 499.39 & C & 11 & 22 & 2 & 0 & 0 & 0 & 0 & 0 & 36.90 & 496.2 \\
\hline 526.73 & W & 14 & 30 & 0 & 0 & 0 & 0 & 0 & 0 & 41.20 & 524.9 \\
\hline 521.59 & $C$ & 13 & 27 & 1 & 0 & 0 & 0 & 0 & 0 & 40.45 & 520.0 \\
\hline 520.59 & $C$ & 13 & 27 & 1 & 0 & 0 & 0 & 0 & 0 & 40.45 & 520.0 \\
\hline 518.99 & C & 12 & 24 & 2 & 0 & 0 & 0 & 0 & 0 & 39.70 & 515.1 \\
\hline 504.10 & $N$ & 11 & 23 & 0 & 0 & 1 & 0 & 0 & 0 & 38.80 & 509.1 \\
\hline 493.00 & $N$ & 8 & 16 & 0 & 0 & 2 & 0 & 0 & 0 & 36.40 & 492.7 \\
\hline 543.84 & W & 15 & 32 & 0 & 0 & 0 & 0 & 0 & 0 & 44.00 & 542.5 \\
\hline 538.19 & C & 14 & 29 & 1 & 0 & 0 & 0 & 0 & 0 & 43.25 & 537.8 \\
\hline 537.19 & C & 14 & 29 & 1 & 0 & 0 & 0 & 0 & 0 & 43.25 & 537.8 \\
\hline 536.79 & $C$ & 13 & 26 & 2 & 0 & 0 & 0 & 0 & 0 & 42.50 & 533.1 \\
\hline 524.75 & $\mathrm{~N}$ & 12 & 25 & 0 & 0 & 1 & 0 & 0 & 0 & 41.60 & 527.4 \\
\hline 519.35 & $\mathrm{~N}$ & 11 & 22 & 1 & 0 & 1 & 0 & 0 & 0 & 40.85 & 522.6 \\
\hline 559.99 & w & 16 & 34 & 0 & 0 & 0 & 0 & 0 & 0 & 46.80 & 559.4 \\
\hline 555.19 & C & 15 & 31 & 1 & 0 & 0 & 0 & 0 & 0 & 46.05 & 554.9 \\
\hline 553.99 & C & 15 & 31 & 1 & 0 & 0 & 0 & 0 & 0 & 46.05 & 554.9 \\
\hline 553.39 & C & 14 & 28 & 2 & 0 & 0 & 0 & 0 & 0 & 45.30 & 550.4 \\
\hline 575.17 & W & 17 & 36 & 0 & 0 & 0 & 0 & 0 & 0 & 49.60 & 575.6 \\
\hline 567.99 & C & 16 & 33 & 1 & 0 & 0 & 0 & 0 & 0 & 48.85 & 571.3 \\
\hline 567.19 & C & 16 & 33 & 1 & 0 & 0 & 0 & 0 & 0 & 48.85 & 571.3 \\
\hline 568.79 & C & 15 & 30 & 2 & 0 & 0 & 0 & 0 & 0 & 48.10 & 567.0 \\
\hline 589.45 & W & 18 & 38 & 0 & 0 & 0 & 0 & 0 & 0 & 52.40 & 591.2 \\
\hline 583.19 & C & 17 & 35 & 1 & 0 & 0 & 0 & 0 & 0 & 51.65 & 587.1 \\
\hline 580.99 & $C$ & 17 & 35 & 1 & 0 & 0 & 0 & 0 & 0 & 51.65 & 587.1 \\
\hline 583.19 & C & 16 & 32 & 2 & 0 & 0 & 0 & 0 & 0 & 50.90 & 582.9 \\
\hline 603.05 & W & 19 & 40 & 0 & 0 & 0 & 0 & 0 & 0 & 55.20 & 606.3 \\
\hline 596.59 & C & 18 & 37 & 1 & 0 & 0 & 0 & 0 & 0 & 54.45 & 602.3 \\
\hline 594.99 & $C$ & 18 & 37 & 1 & 0 & 0 & 0 & 0 & 0 & 54.45 & 602.3 \\
\hline 597.99 & C & 17 & 34 & 2 & 0 & 0 & 0 & 0 & 0 & 53.70 & 598.3 \\
\hline 616.95 & W & 20 & 42 & 0 & 0 & 0 & 0 & 0 & 0 & 58.00 & 620.8 \\
\hline 609.19 & C & 19 & 39 & 1 & 0 & 0 & 0 & 0 & 0 & 57.25 & 617.0 \\
\hline
\end{tabular}




$\begin{array}{ll}183 & \text { 2-methylnonadecane } \\ 184 & \text { 2,3-dimethyloctadecane } \\ 185 & \text { heneicosane } \\ 186 & \text { 2-methyleicosane } \\ 187 & \text { 3-methyleicosane } \\ 188 & \text { 2,3-dimethylnonadecane } \\ 189 & \text { docosane } \\ 190 & \text { 3-methylheneicosane } \\ 191 & \text { tricosane } \\ 192 & \text { tetracosane } \\ 193 & \text { pentacosane } \\ 194 & \text { hexacosane } \\ 195 & \text { heptacosane } \\ 196 & \text { octacosane } \\ 197 & \text { nonacosane } \\ 198 & \text { triacontane }\end{array}$

$\begin{array}{llllllllllll}606.99 & \text { C } & 19 & 39 & 1 & 0 & 0 & 0 & 0 & 0 & 57.25 & 617.0 \\ 611.99 & \text { C } & 18 & 36 & 2 & 0 & 0 & 0 & 0 & 0 & 56.50 & 613.1 \\ 629.65 & \text { W } & 21 & 44 & 0 & 0 & 0 & 0 & 0 & 0 & 60.80 & 635.0 \\ 620.99 & \text { C } & 20 & 41 & 1 & 0 & 0 & 0 & 0 & 0 & 60.05 & 631.2 \\ 620.19 & \text { C } & 20 & 41 & 1 & 0 & 0 & 0 & 0 & 0 & 60.05 & 631.2 \\ 624.99 & \text { C } & 19 & 38 & 2 & 0 & 0 & 0 & 0 & 0 & 59.30 & 627.5 \\ 641.75 & \text { W } & 22 & 46 & 0 & 0 & 0 & 0 & 0 & 0 & 63.60 & 648.6 \\ 631.19 & \text { C } & 21 & 43 & 1 & 0 & 0 & 0 & 0 & 0 & 62.85 & 645.0 \\ 653.25 & \text { W } & 23 & 48 & 0 & 0 & 0 & 0 & 0 & 0 & 66.40 & 661.9 \\ 664.45 & \text { W } & 24 & 50 & 0 & 0 & 0 & 0 & 0 & 0 & 69.20 & 674.8 \\ 675.05 & \text { W } & 25 & 52 & 0 & 0 & 0 & 0 & 0 & 0 & 72.00 & 687.4 \\ 685.35 & \text { W } & 26 & 54 & 0 & 0 & 0 & 0 & 0 & 0 & 74.80 & 699.7 \\ 695.35 & \text { W } & 27 & 56 & 0 & 0 & 0 & 0 & 0 & 0 & 77.60 & 711.6 \\ 704.75 & \text { W } & 28 & 58 & 0 & 0 & 0 & 0 & 0 & 0 & 80.40 & 723.3 \\ 713.95 & \text { W } & 29 & 60 & 0 & 0 & 0 & 0 & 0 & 0 & 83.20 & 734.7 \\ 722.75 & \text { W } & 30 & 62 & 0 & 0 & 0 & 0 & 0 & 0 & 86.00 & 745.8\end{array}$

$\underline{\text { References for literature boiling point data: }}$

C: Cao and Liu (reference 16)

$\mathrm{N}$ : NIST WebBook (reference 17)

W: Wilhoit and Zwolinski (reference 15)

The parameter counts above reflect connectivity and not IUPAC nomenclature. Thus, 2,2,4,4-tetramethylpentane is counted as having two 2,2-dimethyl $(D)$ groups. As an example of the Kinney procedure, consider 3-ethyl-2,2-dimethylhexane. There are six carbons in the main chain, and they bear eleven hydrogen atoms. Also attached to the main chain are two methyl groups and one ethyl group.

The two methyl groups are attached to C2, so there is one $D$ count. Also, the ethyl and two methyl groups are on adjacent carbons, so there is one $V_{2}$ or 3 count. Thus the original Kinney $Y$ value for this compound is

Therefore

$$
Y=0.8 * 6+11+3.05 * 2+5.5-0.4+0.5=27.5
$$

This predicted value differs by about $4.6 \mathrm{~K}$ from the literature boiling point, which is $429.25 \mathrm{~K}$. 
Table 2. Calculation of $Y_{\mathrm{BP}}{ }^{1 / 3}$ (from Equation 6) and $Y_{\mathrm{BP}}$ Values for Compounds in the Training Set.

$\begin{aligned} \text { No. } & \text { Compound } \\ 1 & \text { hexane } \\ 2 & \text { 2,2-dimethylbutane } \\ 3 & \text { 2-methylhexane } \\ 4 & \text { 3-ethylpentane } \\ 5 & \text { 2,3-dimethylpentane } \\ 6 & \text { 2,2-dimethylpentane } \\ 7 & \text { 2,2,3-trimethylbutane } \\ 8 & \text { 4-methylheptane } \\ 9 & \text { 2-methylheptane } \\ 10 & \text { 3,4-dimethylhexane } \\ 11 & \text { 3,3-dimethylhexane } \\ 12 & \text { 2,5-dimethylhexane } \\ 13 & \text { 2,2-dimethylhexane } \\ 14 & \text { 3-ethyl-3-methylpentane } \\ 15 & \text { 3-ethyl-2-methylpentane } \\ 16 & \text { 2,3,3-trimethylpentane } \\ 17 & \text { 2,2,3-trimethylpentane } \\ 18 & \text { 2,2,3,3-tetramethylbutane } \\ 19 & \text { nonane } \\ 20 & \text { 2-methyloctane } \\ 21 & \text { 4-methyloctane } \\ 22 & \text { 3-ethylheptane } \\ 23 & \text { 4-ethylheptane } \\ 24 & \text { 3,3-dimethylheptane } \\ 25 & \text { 3,5-dimethylheptane } \\ 26 & \text { 2,5-dimethylheptane } \\ 27 & \text { 2,6-dimethylheptane } \\ 28 & \text { 3-ethyl-3-methylhexane } \\ 29 & \text { 3-ethyl-4-methylhexane } \\ 30 & \text { 2,3,4-trimethylhexane } \\ 31 & \text { 3-ethyl-2-methylhexane } \\ 32 & \text { 2,3,3-trimethylhexane } \\ 33 & \text { 4-ethyl-2-methylhexane } \\ & \end{aligned}$

$\begin{array}{lll}\text { BP }(\mathbf{K}) & \underline{\boldsymbol{Y}}_{\text {BP }}{ }^{1 / 3} & \underline{\boldsymbol{Y}}_{\text {BP }} \\ 341.89 & 2.665 & 18.93 \\ 322.89 & 2.589 & 17.35 \\ 363.20 & 2.751 & 20.83 \\ 366.63 & 2.765 & 21.15 \\ 362.93 & 2.750 & 20.81 \\ 352.35 & 2.707 & 19.84 \\ 354.03 & 2.714 & 19.99 \\ 390.86 & 2.865 & 23.52 \\ 390.80 & 2.865 & 23.52 \\ 390.38 & 2.863 & 23.47 \\ 385.12 & 2.842 & 22.94 \\ 382.25 & 2.830 & 22.66 \\ 381.99 & 2.829 & 22.63 \\ 391.41 & 2.868 & 23.58 \\ 388.80 & 2.857 & 23.31 \\ 387.91 & 2.853 & 23.22 \\ 382.99 & 2.833 & 22.73 \\ 379.62 & 2.819 & 22.40 \\ 423.95 & 3.004 & 27.10 \\ 416.41 & 2.972 & 26.25 \\ 415.57 & 2.969 & 26.16 \\ 416.15 & 2.971 & 26.22 \\ 414.35 & 2.963 & 26.02 \\ 410.16 & 2.946 & 25.56 \\ 409.15 & 2.942 & 25.45 \\ 409.15 & 2.942 & 25.45 \\ 408.36 & 2.938 & 25.37 \\ 413.75 & 2.961 & 25.96 \\ 413.55 & 2.960 & 25.93 \\ 412.19 & 2.954 & 25.79 \\ 411.15 & 2.950 & 25.67 \\ 410.83 & 2.949 & 25.64 \\ 406.95 & 2.932 & 25.21\end{array}$




\begin{tabular}{|c|c|c|c|c|}
\hline 34 & 2,2,3-trimethylhexane & 406.75 & 2.931 & 25.19 \\
\hline 35 & 2,3,5-trimethylhexane & 404.49 & 2.922 & 24.95 \\
\hline 36 & 2,2,4-trimethylhexane & 399.69 & 2.902 & 24.44 \\
\hline 37 & 3,3-diethylpentane & 419.32 & 2.984 & 26.58 \\
\hline 38 & 3-ethyl-2,3-dimethylpentane & 417.85 & 2.978 & 26.41 \\
\hline 39 & 3-ethyl-2,2-dimethylpentane & 406.98 & 2.932 & 25.22 \\
\hline 40 & 2,2,3,4-tetramethylpentane & 406.17 & 2.929 & 25.13 \\
\hline 41 & decane & 447.27 & 3.103 & 29.88 \\
\hline 42 & 3-methylnonane & 440.95 & 3.076 & 29.11 \\
\hline 43 & 2-methylnonane & 440.15 & 3.073 & 29.01 \\
\hline 44 & 3-ethyloctane & 439.65 & 3.071 & 28.95 \\
\hline 45 & 4-ethyloctane & 436.79 & 3.058 & 28.61 \\
\hline 46 & 3,4-dimethyloctane & 436.55 & 3.057 & 28.58 \\
\hline 47 & 3,5-dimethyloctane & 432.55 & 3.040 & 28.10 \\
\hline 48 & 2,5-dimethyloctane & 431.65 & 3.037 & 28.00 \\
\hline 49 & 4,4-dimethyloctane & 430.65 & 3.032 & 27.88 \\
\hline 50 & 3-ethyl-3-methylheptane & 436.95 & 3.059 & 28.63 \\
\hline 51 & 3,3,4-trimethylheptane & 435.05 & 3.051 & 28.40 \\
\hline 52 & 3-ethyl-2-methylheptane & 434.35 & 3.048 & 28.32 \\
\hline 53 & 4-ethyl-4-methylheptane & 433.95 & 3.046 & 28.27 \\
\hline 54 & 2,3,5-trimethylheptane & 433.85 & 3.046 & 28.26 \\
\hline 55 & 2,3,3-trimethylheptane & 433.35 & 3.044 & 28.20 \\
\hline 56 & 2,3,4-trimethylheptane & 433.05 & 3.042 & 28.16 \\
\hline 57 & 3-ethyl-5-methylheptane & 431.35 & 3.035 & 27.96 \\
\hline 58 & 4-propylheptane & 430.65 & 3.032 & 27.88 \\
\hline 59 & 2,4,5-trimethylheptane & 429.65 & 3.028 & 27.76 \\
\hline 60 & 4-ethyl-2-methylheptane & 429.35 & 3.027 & 27.73 \\
\hline 61 & 2,3,6-trimethylheptane & 429.15 & 3.026 & 27.71 \\
\hline 62 & 3,3,5-trimethylheptane & 428.83 & 3.025 & 27.67 \\
\hline 63 & 2,5,5-trimethylheptane & 425.95 & 3.012 & 27.33 \\
\hline 64 & 2,4,4-trimethylheptane & 424.15 & 3.005 & 27.13 \\
\hline 65 & 2,2,5-trimethylheptane & 423.95 & 3.004 & 27.10 \\
\hline 66 & 2,4,6-trimethylheptane & 420.75 & 2.990 & 26.74 \\
\hline 67 & 3,3,4,4-tetramethylhexane & 443.15 & 3.086 & 29.38 \\
\hline 68 & 2,3,3,4-tetramethylhexane & 437.74 & 3.062 & 28.72 \\
\hline 69 & 3-ethyl-2,3-dimethylhexane & 436.85 & 3.059 & 28.61 \\
\hline 70 & 3-ethyl-2,4-dimethylhexane & 433.25 & 3.043 & 28.19 \\
\hline 71 & 3-ethyl-2,2-dimethylhexane & 429.25 & 3.026 & 27.72 \\
\hline 72 & 3-ethyl-2,5-dimethylhexane & 427.25 & 3.018 & 27.48 \\
\hline 73 & 2,2,4,4-tetramethylhexane & 426.95 & 3.017 & 27.45 \\
\hline
\end{tabular}




\begin{tabular}{rlrrr}
74 & 2,2,3,5-tetramethylhexane & 421.55 & 2.994 & 26.83 \\
75 & 2,2,4,5-tetramethylhexane & 421.03 & 2.992 & 26.77 \\
76 & 4-ethyl-2,2-dimethylhexane & 420.15 & 2.988 & 26.67 \\
77 & 2,2,5,5-tetramethylhexane & 410.61 & 2.948 & 25.61 \\
78 & 3,3-diethyl-2-methylpentane & 442.85 & 3.084 & 29.34 \\
79 & 3-ethyl-2,3,4-trimethylpentane & 442.59 & 3.083 & 29.31 \\
80 & 2,2,3,4,4-pentamethylpentane & 432.44 & 3.040 & 28.09 \\
81 & 3-ethyl-2,2,4-trimethylpentane & 428.45 & 3.023 & 27.62 \\
82 & 3-methyldecane & 463.99 & 3.175 & 32.02 \\
83 & 2-methyldecane & 462.39 & 3.168 & 31.81 \\
84 & 2,3-dimethylnonane & 459.99 & 3.158 & 31.49 \\
85 & dodecane & 489.47 & 3.287 & 35.50 \\
86 & 3-methylundecane & 484.39 & 3.264 & 34.78 \\
87 & 2-methylundecane & 483.39 & 3.260 & 34.64 \\
88 & 2,3-dimethyldecane & 479.99 & 3.245 & 34.17 \\
89 & 2,2-dimethyldecane & 472.19 & 3.211 & 33.11 \\
90 & tridecane & 508.62 & 3.372 & 38.33 \\
91 & 3-methyldodecane & 503.39 & 3.348 & 37.54 \\
92 & 2-methyldodecane & 502.59 & 3.345 & 37.42 \\
93 & 2,3-dimethylundecane & 499.39 & 3.331 & 36.94 \\
94 & tetradecane & 526.73 & 3.453 & 41.17 \\
95 & 3-methyltridecane & 521.59 & 3.430 & 40.34 \\
96 & 2-methyltridecane & 520.59 & 3.425 & 40.19 \\
97 & 2,3-dimethyldodecane & 518.99 & 3.418 & 39.93 \\
98 & 4-propylundecane & 504.10 & 3.351 & 37.64 \\
99 & pentadecane & 543.84 & 3.531 & 44.01 \\
100 & 2-methyltetradecane & 537.19 & 3.500 & 42.89 \\
101 & 4-methyl-6-propylundecane & 519.35 & 3.420 & 39.99 \\
102 & hexadecane & 559.99 & 3.605 & 46.85 \\
103 & 3-methylpentadecane & 555.19 & 3.583 & 45.99 \\
104 & 2,3-dimethyltetradecane & 553.39 & 3.574 & 45.67 \\
105 & heptadecane & 575.17 & 3.676 & 49.65 \\
106 & 2-methylhexadecane & 567.99 & 3.642 & 48.31 \\
107 & 3-methylhexadecane & 567.19 & 3.638 & 48.16 \\
108 & octadecane & 589.45 & 3.743 & 52.42 \\
109 & 3-methylheptadecane & 583.19 & 3.713 & 51.19 \\
110 & 2-methylheptadecane & 580.99 & 3.703 & 50.77 \\
111 & 2,3-dimethylhexadecane & 583.19 & 3.713 & 51.19 \\
112 & nonadecane & 603.05 & 3.807 & 55.19 \\
113 & 3-methyloctadecane & 596.59 & 3.776 & 53.86 \\
& & & & \\
\hline
\end{tabular}


2-methyleicosane

620.99

119 3-methyleicosane

$620.19 \quad 3.890 \quad 58.85$

120 2,3-dimethylnonadecane

$624.99 \quad 3.913 \quad 59.91$

121 3-methylheneicosane

$631.19 \quad 3.943 \quad 61.31$

tetracosane

$\begin{array}{lll}664.45 & 4.108 & 69.31\end{array}$

pentacosane

$\begin{array}{lll}675.05 & 4.161 & 72.05\end{array}$

hexacosane

$\begin{array}{lll}685.35 & 4.213 \quad 74.80\end{array}$

125 heptacosane

$\begin{array}{lll}695.35 & 4.265 & 77.57\end{array}$

126

nonacosane

713.95

$4.361 \quad 82.96$ 
Table 3. Parameters Used in Equation 7, $Y_{R}$ Values Predicted with Equation 7, Literature Boiling

Points, and Boiling Points Predicted with Equation 5 for the Compounds in the Training Set.

No. Compound

1 hexane

2 2,2-dimethylbutane

3 2-methylhexane

4 3-ethylpentane

5 2,3-dimethylpentane

6 2,2-dimethylpentane

7 2,2,3-trimethylbutane

8 4-methylheptane

9 2-methylheptane

10 3,4-dimethylhexane

11 3,3-dimethylhexane

12 2,5-dimethylhexane

13 2,2-dimethylhexane

14 3-ethyl-3-methylpentane

15 3-ethyl-2-methylpentane

16 2,3,3-trimethylpentane

17 2,2,3-trimethylpentane

18 2,2,3,3-tetramethylbutane

19 nonane

20 2-methyloctane

21 4-methyloctane

22 3-ethylheptane

23 4-ethylheptane

24 3,3-dimethylheptane

25 3,5-dimethylheptane

26 2,5-dimethylheptane

27 2,6-dimethylheptane

28 3-ethyl-3-methylhexane

29 3-ethyl-4-methylhexane

30 2,3,4-trimethylhexane

31 3-ethyl-2-methylhexane

32 2,3,3-trimethylhexane

33 4-ethyl-2-methylhexane

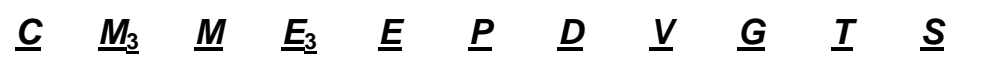

$\underline{Y_{R}} \quad \underline{B P(K)}$

Pred.

BP (K) BP (K)

$\begin{array}{llllll}6 & 0 & 0 & 0 & 0 & 0 \\ 4 & 2 & 0 & 0 & 0 & 0 \\ 6 & 0 & 1 & 0 & 0 & 0 \\ 5 & 0 & 0 & 1 & 0 & 0 \\ 5 & 1 & 1 & 0 & 0 & 0 \\ 5 & 0 & 2 & 0 & 0 & 0 \\ 4 & 3 & 0 & 0 & 0 & 0 \\ 7 & 0 & 1 & 0 & 0 & 0 \\ 7 & 0 & 1 & 0 & 0 & 0 \\ 6 & 2 & 0 & 0 & 0 & 0 \\ 6 & 2 & 0 & 0 & 0 & 0 \\ 6 & 0 & 2 & 0 & 0 & 0 \\ 6 & 0 & 2 & 0 & 0 & 0 \\ 5 & 1 & 0 & 1 & 0 & 0 \\ 5 & 0 & 1 & 1 & 0 & 0 \\ 5 & 2 & 1 & 0 & 0 & 0 \\ 5 & 1 & 2 & 0 & 0 & 0 \\ 4 & 0 & 4 & 0 & 0 & 0 \\ 9 & 0 & 0 & 0 & 0 & 0 \\ 8 & 0 & 1 & 0 & 0 & 0 \\ 8 & 0 & 1 & 0 & 0 & 0 \\ 7 & 0 & 0 & 1 & 0 & 0 \\ 7 & 0 & 0 & 0 & 1 & 0 \\ 7 & 2 & 0 & 0 & 0 & 0 \\ 7 & 2 & 0 & 0 & 0 & 0 \\ 7 & 1 & 1 & 0 & 0 & 0 \\ 7 & 0 & 2 & 0 & 0 & 0 \\ 6 & 1 & 0 & 1 & 0 & 0 \\ 6 & 1 & 0 & 1 & 0 & 0 \\ 6 & 2 & 1 & 0 & 0 & 0 \\ 6 & 0 & 1 & 1 & 0 & 0 \\ 6 & 2 & 1 & 0 & 0 & 0 \\ 6 & 0 & 1 & 0 & 1 & 0 \\ & & & & \end{array}$

$\begin{array}{rrr}18.97 & 341.89 & 342.42 \\ 17.23 & 322.89 & 321.47 \\ 20.74 & 363.2 & 362.26 \\ 20.95 & 366.63 & 364.47 \\ 20.50 & 362.93 & 359.65 \\ 19.54 & 352.35 & 348.96 \\ 20.37 & 354.03 & 358.24 \\ 23.49 & 390.86 & 390.53 \\ 23.49 & 390.80 & 390.53 \\ 23.33 & 390.38 & 388.95 \\ 23.09 & 385.12 & 386.59 \\ 22.55 & 382.25 & 381.13 \\ 22.22 & 381.99 & 377.77 \\ 23.25 & 391.41 & 388.16 \\ 23.34 & 388.80 & 389.01 \\ 23.28 & 387.91 & 388.49 \\ 22.56 & 382.99 & 381.28 \\ 22.65 & 379.62 & 382.18 \\ 27.31 & 423.95 & 425.72 \\ 26.25 & 416.41 & 416.35 \\ 26.25 & 415.57 & 416.35 \\ 26.33 & 416.15 & 417.10 \\ 26.03 & 414.35 & 414.41 \\ 25.80 & 410.16 & 412.30 \\ 25.56 & 409.15 & 410.11 \\ 25.41 & 409.15 & 408.71 \\ 25.25 & 408.36 & 407.31 \\ 25.85 & 413.75 & 412.78 \\ 26.09 & 413.55 & 414.93 \\ 25.64 & 412.19 & 410.89 \\ 25.94 & 411.15 & 413.56 \\ 25.88 & 410.83 & 413.08 \\ 25.16 & 406.95 & 406.43\end{array}$




\begin{tabular}{|c|c|}
\hline 34 & 2,2,3-trimethylhexane \\
\hline 35 & 2,3,5-trimethylhexane \\
\hline 36 & 2,2,4-trimethylhexane \\
\hline 37 & 3,3-diethylpentane \\
\hline 38 & 3-ethyl-2,3-dimethylpentane \\
\hline 39 & 3-ethyl-2,2-dimethylpentane \\
\hline 40 & 2,2,3,4-tetramethylpentane \\
\hline 41 & decane \\
\hline 42 & 3-methylnonane \\
\hline 43 & 2-methylnonane \\
\hline 44 & 3-ethyloctane \\
\hline 45 & 4-ethyloctane \\
\hline 46 & 3,4-dimethyloctane \\
\hline 47 & 3,5-dimethyloctane \\
\hline 48 & 2,5-dimethyloctane \\
\hline 49 & 4,4-dimethyloctane \\
\hline 50 & 3-ethyl-3-methylheptane \\
\hline 51 & 3,3,4-trimethylheptane \\
\hline 52 & 3-ethyl-2-methylheptane \\
\hline 53 & 4-ethyl-4-methylheptane \\
\hline 54 & 2,3,5-trimethylheptane \\
\hline 55 & 2,3,3-trimethylheptane \\
\hline 56 & 2,3,4-trimethylheptane \\
\hline 57 & 3-ethyl-5-methylheptane \\
\hline 58 & 4-propylheptane \\
\hline 59 & 2,4,5-trimethylheptane \\
\hline 60 & 4-ethyl-2-methylheptane \\
\hline 61 & 2,3,6-trimethylheptane \\
\hline 62 & 3,3,5-trimethylheptane \\
\hline 63 & 2,5,5-trimethylheptane \\
\hline 64 & 2,4,4-trimethylheptane \\
\hline 65 & 2,2,5-trimethylheptane \\
\hline 66 & 2,4,6-trimethylheptane \\
\hline 67 & 3,3,4,4-tetramethylhexane \\
\hline 68 & 2,3,3,4-tetramethylhexane \\
\hline 69 & 3-ethyl-2,3-dimethylhexane \\
\hline 70 & 3-ethyl-2,4-dimethylhexane \\
\hline 71 & 3-ethyl-2,2-dimethylhexane \\
\hline 72 & 3-ethyl-2,5-dimethylhexane \\
\hline 73 & 2,2,4,4-tetramethylhexane \\
\hline 74 & 2,2,3,5-tetramethylhexane \\
\hline
\end{tabular}

\begin{tabular}{|c|c|c|c|c|c|c|c|c|c|c|c|c|c|}
\hline 6 & 1 & 2 & 0 & 0 & 0 & 1 & 2 & 0 & 0 & 2.25 & 25.16 & 406.75 & 406.47 \\
\hline 6 & 1 & 2 & 0 & 0 & 0 & 0 & 1 & 0 & 0 & 2.25 & 25.01 & 404.49 & 405.07 \\
\hline 6 & 1 & 2 & 0 & 0 & 0 & 1 & 0 & 0 & 0 & 2.25 & 24.20 & 399.69 & 397.44 \\
\hline 5 & 0 & 0 & 2 & 0 & 0 & 0 & 0 & 1 & 0 & 3.24 & 26.13 & 419.32 & 415.28 \\
\hline 5 & 1 & 1 & 1 & 0 & 0 & 0 & 2 & 1 & 0 & 3.24 & 26.16 & 417.85 & 415.59 \\
\hline 5 & 0 & 2 & 1 & 0 & 0 & 1 & 2 & 0 & 0 & 3.24 & 25.44 & 406.98 & 409.03 \\
\hline 5 & 1 & 3 & 0 & 0 & 0 & 1 & 3 & 0 & 0 & 3.24 & 24.99 & 406.17 & 404.91 \\
\hline 10 & 0 & 0 & 0 & 0 & 0 & 0 & 0 & 0 & 0 & 1.00 & 30.09 & 447.27 & 448.90 \\
\hline 9 & 1 & 0 & 0 & 0 & 0 & 0 & 0 & 0 & 0 & 1.23 & 29.16 & 440.95 & 441.36 \\
\hline 9 & 0 & 1 & 0 & 0 & 0 & 0 & 0 & 0 & 0 & 1.23 & 29.01 & 440.15 & 440.11 \\
\hline 8 & 0 & 0 & 1 & 0 & 0 & 0 & 0 & 0 & 0 & 1.56 & 29.06 & 439.65 & 440.52 \\
\hline 8 & 0 & 0 & 0 & 1 & 0 & 0 & 0 & 0 & 0 & 1.56 & 28.76 & 436.79 & 438.04 \\
\hline 8 & 1 & 1 & 0 & 0 & 0 & 0 & 1 & 0 & 0 & 1.56 & 28.61 & 436.55 & 436.82 \\
\hline 8 & 1 & 1 & 0 & 0 & 0 & 0 & 0 & 0 & 0 & 1.56 & 28.13 & 432.55 & 432.79 \\
\hline 8 & 0 & 2 & 0 & 0 & 0 & 0 & 0 & 0 & 0 & 1.56 & 27.98 & 431.65 & 431.50 \\
\hline 8 & 0 & 2 & 0 & 0 & 0 & 0 & 0 & 1 & 0 & 1.56 & 28.22 & 430.65 & 433.54 \\
\hline 7 & 1 & 0 & 1 & 0 & 0 & 0 & 0 & 1 & 0 & 2.04 & 28.51 & 436.95 & 435.96 \\
\hline 7 & 2 & 1 & 0 & 0 & 0 & 0 & 2 & 1 & 0 & 2.04 & 28.54 & 435.05 & 436.24 \\
\hline 7 & 0 & 1 & 1 & 0 & 0 & 0 & 1 & 0 & 0 & 2.04 & 28.59 & 434.35 & 436.69 \\
\hline 7 & 0 & 1 & 0 & 1 & 0 & 0 & 0 & 1 & 0 & 2.04 & 28.06 & 433.95 & 432.16 \\
\hline 7 & 2 & 1 & 0 & 0 & 0 & 0 & 1 & 0 & 0 & 2.04 & 27.82 & 433.85 & 430.15 \\
\hline 7 & 2 & 1 & 0 & 0 & 0 & 0 & 2 & 1 & 0 & 2.04 & 28.54 & 433.35 & 436.24 \\
\hline 7 & 1 & 2 & 0 & 0 & 0 & 0 & 2 & 0 & 0 & 2.04 & 28.15 & 433.05 & 432.94 \\
\hline 7 & 1 & 0 & 1 & 0 & 0 & 0 & 0 & 0 & 0 & 2.04 & 28.27 & 431.35 & 433.93 \\
\hline 7 & 0 & 0 & 0 & 0 & 1 & 0 & 0 & 0 & 0 & 2.04 & 27.36 & 430.65 & 426.13 \\
\hline 7 & 1 & 2 & 0 & 0 & 0 & 0 & 1 & 0 & 0 & 2.04 & 27.67 & 429.65 & 428.85 \\
\hline 7 & 0 & 1 & 0 & 1 & 0 & 0 & 0 & 0 & 0 & 2.04 & 27.82 & 429.35 & 430.10 \\
\hline 7 & 1 & 2 & 0 & 0 & 0 & 0 & 1 & 0 & 0 & 2.04 & 27.67 & 429.15 & 428.85 \\
\hline 7 & 3 & 0 & 0 & 0 & 0 & 0 & 0 & 1 & 0 & 2.04 & 27.74 & 428.83 & 429.41 \\
\hline 7 & 2 & 1 & 0 & 0 & 0 & 0 & 0 & 1 & 0 & 2.04 & 27.58 & 425.95 & 428.11 \\
\hline 7 & 0 & 3 & 0 & 0 & 0 & 0 & 0 & 1 & 0 & 2.04 & 27.28 & 424.15 & 425.48 \\
\hline 7 & 1 & 2 & 0 & 0 & 0 & 1 & 0 & 0 & 0 & 2.04 & 26.86 & 423.95 & 421.82 \\
\hline 7 & 0 & 3 & 0 & 0 & 0 & 0 & 0 & 0 & 0 & 2.04 & 27.04 & 420.75 & 423.38 \\
\hline 6 & 4 & 0 & 0 & 0 & 0 & 0 & 4 & 2 & 0 & 2.78 & 29.25 & 443.15 & 442.14 \\
\hline 6 & 3 & 1 & 0 & 0 & 0 & 0 & 4 & 1 & 0 & 2.78 & 28.86 & 437.74 & 438.90 \\
\hline 6 & 1 & 1 & 1 & 0 & 0 & 0 & 2 & 1 & 0 & 2.78 & 28.67 & 436.85 & 437.35 \\
\hline 6 & 1 & 1 & 1 & 0 & 0 & 0 & 2 & 0 & 0 & 2.78 & 28.43 & 433.25 & 435.34 \\
\hline 6 & 0 & 2 & 1 & 0 & 0 & 1 & 2 & 0 & 0 & 2.78 & 27.95 & 429.25 & 431.27 \\
\hline 6 & 0 & 2 & 1 & 0 & 0 & 0 & 1 & 0 & 0 & 2.78 & 27.80 & 427.25 & 429.99 \\
\hline 6 & 2 & 2 & 0 & 0 & 0 & 1 & 0 & 1 & 1 & 2.78 & 27.43 & 426.95 & 426.78 \\
\hline 6 & 1 & 3 & 0 & 0 & 0 & 1 & 2 & 0 & 0 & 2.78 & 27.03 & 421.55 & 423.28 \\
\hline
\end{tabular}




\begin{tabular}{|c|c|}
\hline 75 & 2,2,4,5-tetramethylhexane \\
\hline 76 & 4-ethyl-2,2-dimethylhexane \\
\hline 77 & 2,2,5,5-tetramethylhexane \\
\hline 78 & 3,3-diethyl-2-methylpentane \\
\hline 79 & 3-ethyl-2,3,4-trimethylpentane \\
\hline 80 & 2,2,3,4,4-pentamethylpentane \\
\hline 81 & 3-ethyl-2,2,4-trimethylpentane \\
\hline 82 & 3-methyldecane \\
\hline 83 & 2-methyldecane \\
\hline 84 & 2,3-dimethylnonane \\
\hline 85 & dodecane \\
\hline 86 & 3-methylundecane \\
\hline 87 & 2-methylundecane \\
\hline 88 & 2,3-dimethyldecane \\
\hline 89 & 2,2-dimethyldecane \\
\hline 90 & tridecane \\
\hline 91 & 3-methyldodecane \\
\hline 92 & 2-methyldodecane \\
\hline 93 & 2,3-dimethylundecane \\
\hline 94 & tetradecane \\
\hline 95 & 3-methyltridecane \\
\hline 96 & 2-methyltridecane \\
\hline 97 & 2,3-dimethyldodecane \\
\hline 98 & 4-propylundecane \\
\hline 99 & pentadecane \\
\hline 100 & 2-methyltetradecane \\
\hline 101 & 4-methyl-6-propylundecane \\
\hline 102 & hexadecane \\
\hline 103 & 3-methylpentadecane \\
\hline 104 & 2,3-dimethyltetradecane \\
\hline 105 & heptadecane \\
\hline 106 & 2-methylhexadecane \\
\hline 107 & 3-methylhexadecane \\
\hline 108 & octadecane \\
\hline 109 & 3-methylheptadecane \\
\hline 110 & 2-methylheptadecane \\
\hline 111 & 2,3-dimethylhexadecane \\
\hline 112 & nonadecane \\
\hline 113 & 3-methyloctadecane \\
\hline 114 & 2-methyloctadecane \\
\hline 115 & 2,3-dimethylheptadecane \\
\hline
\end{tabular}

\begin{tabular}{|c|c|c|c|}
\hline 6 & 1 & 3 & 0 \\
\hline 6 & 0 & 2 & 0 \\
\hline 6 & 0 & 4 & 0 \\
\hline 5 & 0 & 1 & 2 \\
\hline 5 & 1 & 2 & 1 \\
\hline 5 & 1 & 4 & 0 \\
\hline 5 & 0 & 3 & 1 \\
\hline 10 & 1 & 0 & 0 \\
\hline 10 & 0 & 1 & 0 \\
\hline 9 & 1 & 1 & 0 \\
\hline 12 & 0 & 0 & 0 \\
\hline 11 & 1 & 0 & 0 \\
\hline 11 & 0 & 1 & 0 \\
\hline 10 & 1 & 1 & 0 \\
\hline 10 & 0 & 2 & 0 \\
\hline 13 & 0 & 0 & 0 \\
\hline 12 & 1 & 0 & 0 \\
\hline 12 & 0 & 1 & 0 \\
\hline 11 & 1 & 1 & 0 \\
\hline 14 & 0 & 0 & 0 \\
\hline 13 & 1 & 0 & 0 \\
\hline 13 & 0 & 1 & 0 \\
\hline 12 & 1 & 1 & 0 \\
\hline 11 & 0 & 0 & 0 \\
\hline 15 & 0 & 0 & 0 \\
\hline 14 & 0 & 1 & 0 \\
\hline 11 & 0 & 1 & 0 \\
\hline 16 & 0 & 0 & 0 \\
\hline 15 & 1 & 0 & 0 \\
\hline 14 & 1 & 1 & 0 \\
\hline 17 & 0 & 0 & 0 \\
\hline 16 & 0 & 1 & 0 \\
\hline 16 & 1 & 0 & 0 \\
\hline 18 & 0 & 0 & 0 \\
\hline 17 & 1 & 0 & 0 \\
\hline 17 & 0 & 1 & 0 \\
\hline 16 & 1 & 1 & 0 \\
\hline 19 & 0 & 0 & 0 \\
\hline 18 & 1 & 0 & 0 \\
\hline 18 & 0 & 1 & 0 \\
\hline 17 & 1 & 1 & 0 \\
\hline
\end{tabular}

O

$\begin{array}{lllll}1 & 1 & 0 & 0 & 2.78 \\ 1 & 0 & 0 & 0 & 2.78 \\ 2 & 0 & 0 & 0 & 2.78 \\ 0 & 2 & 1 & 0 & 4.00 \\ 0 & 4 & 1 & 0 & 4.00 \\ 2 & 4 & 0 & 1 & 4.00 \\ 1 & 3 & 0 & 0 & 4.00 \\ 0 & 0 & 0 & 0 & 1.21 \\ 0 & 0 & 0 & 0 & 1.21 \\ 0 & 1 & 0 & 0 & 1.49 \\ 0 & 0 & 0 & 0 & 1.00 \\ 0 & 0 & 0 & 0 & 1.19 \\ 0 & 0 & 0 & 0 & 1.19 \\ 0 & 1 & 0 & 0 & 1.44 \\ 1 & 0 & 0 & 0 & 1.44 \\ 0 & 0 & 0 & 0 & 1.00 \\ 0 & 0 & 0 & 0 & 1.17 \\ 0 & 0 & 0 & 0 & 1.17 \\ 0 & 1 & 0 & 0 & 1.40 \\ 0 & 0 & 0 & 0 & 1.00 \\ 0 & 0 & 0 & 0 & 1.16 \\ 0 & 0 & 0 & 0 & 1.16 \\ 0 & 1 & 0 & 0 & 1.36 \\ 0 & 0 & 0 & 0 & 1.62 \\ 0 & 0 & 0 & 0 & 1.00 \\ 0 & 0 & 0 & 0 & 1.15 \\ 0 & 0 & 0 & 0 & 1.86 \\ 0 & 0 & 0 & 0 & 1.00 \\ 0 & 0 & 0 & 0 & 1.14 \\ 0 & 1 & 0 & 0 & 1.31 \\ 0 & 0 & 0 & 0 & 1.00 \\ 0 & 0 & 0 & 0 & 1.13 \\ 0 & 0 & 0 & 0 & 1.13 \\ 0 & 0 & 0 & 0 & 1.00 \\ 0 & 0 & 0 & 0 & 1.12 \\ 0 & 0 & 0 & 0 & 1.12 \\ 0 & 1 & 0 & 0 & 1.27 \\ 0 & 0 & 0 & 1.00 \\ 0 & 0 & 0 & 1.11 \\ 0 & 0 & 0 & 1.11 \\ 0 & & & \\ 0 & 0 & 1.25\end{array}$

$26.55 \quad 421.03$

419.06

$26.70 \quad 420.15 \quad 420.35$

$25.59 \quad 410.61 \quad 410.40$

$29.09 \quad 442.85 \quad 440.76$

$29.12 \quad 442.59 \quad 441.04$

$28.11 \quad 432.44 \quad 432.61$

$\begin{array}{lll}27.92 & 428.45 \quad 430.97\end{array}$

$31.92 \quad 463.99 \quad 463.28$

$\begin{array}{lll}31.77 & 462.39 \quad 462.12\end{array}$

$\begin{array}{lll}31.35 & 459.99 \quad 458.87\end{array}$

$35.64 \quad 489.47 \quad 490.45$

$34.69 \quad 484.39 \quad 483.72$

$34.54 \quad 483.39 \quad 482.64$

$34.10 \quad 479.99 \quad 479.46$

$33.14 \quad 472.19 \quad 472.43$

$38.42 \quad 508.62 \quad 509.25$

$37.46 \quad 503.39 \quad 502.86$

$37.31 \quad 502.59 \quad 501.85$

$\begin{array}{lll}36.85 & 499.39 \quad 498.77\end{array}$

$41.20 \quad 526.73 \quad 526.94$

$40.23 \quad 521.59 \quad 520.87$

$40.08 \quad 520.59 \quad 519.91$

$39.61 \quad 518.99 \quad 516.94$

$38.23 \quad 504.10 \quad 507.97$

$43.98 \quad 543.84 \quad 543.66$

$\begin{array}{lll}42.85 & 537.19 \quad 536.97\end{array}$

$39.93 \quad 519.35 \quad 518.97$

$46.76 \quad 559.99 \quad 559.50$

$45.77 \quad 555.19 \quad 553.98$

$45.14 \quad 553.39 \quad 550.35$

$49.54 \quad 575.17 \quad 574.56$

$48.40 \quad 567.99 \quad 568.46$

$48.55 \quad 567.19 \quad 569.28$

$52.32 \quad 589.45 \quad 588.91$

$51.32 \quad 583.19 \quad 583.85$

$\begin{array}{lll}51.17 & 580.99 & 583.07\end{array}$

$50.67 \quad 583.19 \quad 580.49$

$55.09 \quad 603.05 \quad 602.61$

$54.10 \quad 596.59 \quad 597.76$

$53.95 \quad 594.99 \quad 597.02$

$53.44 \quad 597.99 \quad 594.52$ 
116 2-methylnonadecane

117 heneicosane

118 2-methyleicosane

119 3-methyleicosane

120 2,3-dimethylnonadecane

121 3-methylheneicosane

122 tetracosane

123 pentacosane

124 hexacosane

125 heptacosane

126

$\begin{array}{lllll}19 & 0 & 1 & 0 & 0 \\ 21 & 0 & 0 & 0 & 0 \\ 20 & 0 & 1 & 0 & 0 \\ 20 & 1 & 0 & 0 & 0 \\ 19 & 1 & 1 & 0 & 0 \\ 21 & 1 & 0 & 0 & 0 \\ 24 & 0 & 0 & 0 & 0 \\ 25 & 0 & 0 & 0 & 0 \\ 26 & 0 & 0 & 0 & 0 \\ 27 & 0 & 0 & 0 & 0 \\ 29 & 0 & 0 & 0 & 0\end{array}$

0
0
0
0
0
0
0
0
0
0
0

0
0
0
0
1
0
0
0
0
0
0

$\begin{array}{ll}0 & 1.11 \\ 0 & 1.00 \\ 0 & 1.10 \\ 0 & 1.10 \\ 0 & 1.22 \\ 0 & 1.10 \\ 0 & 1.00 \\ 0 & 1.00 \\ 0 & 1.00 \\ 0 & 1.00 \\ 0 & 1.00\end{array}$

$56.72 \quad 606.99$

60.65629 .65

$59.50 \quad 620.99$

$59.65 \quad 620.19$

$58.98 \quad 624.99$

$62.42 \quad 631.19$

$68.99 \quad 664.45$

$71.77 \quad 675.05$

$74.55 \quad 685.35$

$77.32 \quad 695.35$

$82.88 \quad 713.95$

These parameter counts are based on structural relationships, not IUPAC nomenclature. For example, 3,4dimethylhexane is counted as having two 3-methyl groups. Also, the parameter $V$ represents adjacencies, not substituents. Thus $V$ is 4 for 2,2,3,3-tetramethylhexane, 4 for 2,3,3,4-tetramethylpentane, and 3 for 2,3,4,5tetramethylhexane. 


\section{Statistical Parameters for Equation 7.}

$$
\begin{gathered}
Y_{\mathrm{R}}=(1.726 \pm 0.205)+(2.779 \pm 0.007) C+(1.716 \pm 0.064) M_{3}+(1.564 \pm 0.060) M+(4.204 \pm 0.155) E_{3}+ \\
(3.905 \pm 0.168) E+(5.008 \pm 0.208) P-(0.329 \pm 0.105) D+(0.241 \pm 0.088) G+(0.479 \pm 0.046) V \\
+0.967 \pm 0.229) T+(0.574 \pm 0.143) S
\end{gathered}
$$

As an example of the calculation of $Y_{R}$ values, consider 2,2,4,4-tetramethylhexane. There are six carbons on the main chain, so $C$ is 6 . Two methyl substituents on carbon 4 are counted as $M_{3}$ groups because $C 4$ is the third carbon from one end of the chain. Because there are two methyl groups on $\mathrm{C} 2$, the $D$ count is 1 . The two methyl groups on $\mathrm{C} 4$ are geminal, so $G$ is 1 . (The two methyl groups on $\mathrm{C} 2$ are counted as $D$ but not as $G$.) The $-\mathrm{C}\left(\mathrm{CH}_{3}\right)_{2}-\mathrm{CH}_{2}-\mathrm{C}\left(\mathrm{CH}_{3}\right)_{2}-$ grouping gives a $T$ count of 1 . The shape parameter, $S$, is $(10 / 6)^{2}=2.778$. Thus

$$
Y_{\mathrm{R}}=1.726+2.779 * 6+1.716 * 2+1.564 * 2-0.329+0.241+0.967+0.574 * 2.778=27.43
$$

The boiling point predicted with equation 5 is therefore:

$$
B P(\mathrm{~K})=-16.802 *(27.43)^{2 / 3}+337.377 *(27.43)^{1 / 3}-437.884=426.78 \mathrm{~K}
$$

This result compares quite well with the literature boiling point, which is $426.95 \mathrm{~K}$. 
Table 4. Parameter Counts, Predicted $Y_{R}$ Values, and Literature and Predicted Boiling Points for the Test Set.

\section{No. Compound}

$\begin{aligned} 1 & \text { 3-methylpentane } \\ 2 & \text { 2-methylpentane } \\ 3 & \text { 2,3-dimethylbutane } \\ 4 & \text { heptane } \\ 5 & \text { 3-methylhexane } \\ 6 & \text { 3,3-dimethylpentane } \\ 7 & \text { 2,4-dimethylpentane } \\ 8 & \text { octane } \\ 9 & \text { 3-methylheptane } \\ 10 & \text { 3-ethylhexane } \\ 11 & \text { 2,3-dimethylhexane } \\ 12 & \text { 2,4-dimethylhexane } \\ 13 & \text { 2,3,4-trimethylpentane } \\ 14 & \text { 2,2,4-trimethylpentane } \\ 15 & \text { 3-methyloctane } \\ 16 & \text { 3,4-dimethylheptane } \\ 17 & \text { 2,3-dimethylheptane } \\ 18 & \text { 4,4-dimethylheptane } \\ 19 & \text { 2,4-dimethylheptane } \\ 20 & \text { 2,2-dimethylheptane } \\ 21 & \text { 3,3,4-trimethylhexane } \\ 22 & \text { 2,4,4-trimethylhexane } \\ 23 & \text { 2,2,5-trimethylhexane } \\ 24 & \text { 2,3,3,4-tetramethylpentane } \\ 25 & \text { 2,2,3,3-tetramethylpentane } \\ 26 & \text { 3-ethyl-2,4-dimethylpentane } \\ 27 & \text { 2,2,4,4-tetramethylpentane } \\ 28 & \text { 4-methylnonane } \\ 29 & \text { 5-methylnonane } \\ 30 & \text { 2,3-dimethyloctane } \\ 31 & \text { 4,5-dimethyloctane } \\ 32 & \text { 3,3-dimethyloctane } \\ 34 & \text { 3,6-dimethyloctane } \\ & \text { 2,7-dimethyloctane } \\ & \\ 33 & \\ 13 & \end{aligned}$

\begin{tabular}{|c|c|c|c|c|c|c|c|c|c|c|c|c|c|}
\hline$\underline{C}$ & $\underline{M_{3}}$ & $\underline{M}$ & $\underline{E}_{\underline{3}}$ & $\underline{E}$ & $\underline{P}$ & $\underline{D}$ & $\underline{v}$ & $\underline{\mathbf{G}}$ & $\underline{I}$ & $\underline{S}$ & $\underline{Y}_{R}$ & $\begin{array}{c}\text { Lit. } \\
\text { BP (K) }\end{array}$ & $\begin{array}{c}\text { Pred. } \\
\text { BP (K) }\end{array}$ \\
\hline 5 & 1 & 0 & 0 & 0 & 0 & 0 & 0 & 0 & 0 & 1.44 & 18.16 & 336.43 & 332.84 \\
\hline 5 & 0 & 1 & 0 & 0 & 0 & 0 & 0 & 0 & 0 & 1.44 & 18.01 & 333.42 & 331.01 \\
\hline 4 & 2 & 0 & 0 & 0 & 0 & 0 & 1 & 0 & 0 & 2.25 & 18.04 & 331.14 & 331.41 \\
\hline 7 & 0 & 0 & 0 & 0 & 0 & 0 & 0 & 0 & 0 & 1.00 & 21.75 & 371.58 & 372.96 \\
\hline 6 & 1 & 0 & 0 & 0 & 0 & 0 & 0 & 0 & 0 & 1.36 & 20.89 & 365.00 & 363.90 \\
\hline 5 & 2 & 0 & 0 & 0 & 0 & 0 & 0 & 1 & 0 & 1.96 & 20.42 & 359.21 & 358.70 \\
\hline 5 & 0 & 2 & 0 & 0 & 0 & 0 & 0 & 0 & 0 & 1.96 & 19.87 & 353.65 & 352.68 \\
\hline 8 & 0 & 0 & 0 & 0 & 0 & 0 & 0 & 0 & 0 & 1.00 & 24.53 & 398.82 & 400.54 \\
\hline 7 & 1 & 0 & 0 & 0 & 0 & 0 & 0 & 0 & 0 & 1.31 & 23.64 & 392.08 & 392.02 \\
\hline 6 & 0 & 0 & 1 & 0 & 0 & 0 & 0 & 0 & 0 & 1.78 & 23.62 & 391.68 & 391.82 \\
\hline 6 & 1 & 1 & 0 & 0 & 0 & 0 & 1 & 0 & 0 & 1.78 & 23.18 & 388.76 & 387.45 \\
\hline 6 & 1 & 1 & 0 & 0 & 0 & 0 & 0 & 0 & 0 & 1.78 & 22.70 & 382.58 & 382.66 \\
\hline 5 & 1 & 2 & 0 & 0 & 0 & 0 & 2 & 0 & 0 & 2.56 & 22.89 & 386.62 & 384.59 \\
\hline 5 & 0 & 3 & 0 & 0 & 0 & 1 & 0 & 0 & 0 & 2.56 & 21.45 & 372.39 & 369.82 \\
\hline 8 & 1 & 0 & 0 & 0 & 0 & 0 & 0 & 0 & 0 & 1.27 & 26.40 & 417.36 & 417.71 \\
\hline 7 & 1 & 1 & 0 & 0 & 0 & 0 & 1 & 0 & 0 & 1.65 & 25.88 & 413.75 & 413.09 \\
\hline 7 & 1 & 1 & 0 & 0 & 0 & 0 & 1 & 0 & 0 & 1.65 & 25.88 & 413.65 & 413.09 \\
\hline 7 & 0 & 2 & 0 & 0 & 0 & 0 & 0 & 1 & 0 & 1.65 & 25.49 & 408.35 & 409.53 \\
\hline 7 & 0 & 2 & 0 & 0 & 0 & 0 & 0 & 0 & 0 & 1.65 & 25.25 & 406.04 & 407.31 \\
\hline 7 & 0 & 2 & 0 & 0 & 0 & 1 & 0 & 0 & 0 & 1.65 & 24.92 & 405.84 & 404.25 \\
\hline 6 & 3 & 0 & 0 & 0 & 0 & 0 & 2 & 1 & 0 & 2.25 & 26.04 & 413.61 & 414.46 \\
\hline 6 & 2 & 1 & 0 & 0 & 0 & 0 & 0 & 0 & 0 & 2.25 & 24.68 & 403.80 & 402.01 \\
\hline 6 & 0 & 3 & 0 & 0 & 0 & 1 & 0 & 0 & 0 & 2.25 & 24.05 & 397.23 & 395.98 \\
\hline 5 & 2 & 2 & 0 & 0 & 0 & 0 & 4 & 1 & 0 & 3.24 & 26.20 & 414.70 & 415.90 \\
\hline 5 & 2 & 2 & 0 & 0 & 0 & 1 & 4 & 1 & 0 & 3.24 & 25.87 & 413.42 & 412.92 \\
\hline 5 & 0 & 2 & 1 & 0 & 0 & 0 & 2 & 0 & 0 & 3.24 & 25.77 & 409.84 & 412.04 \\
\hline 5 & 0 & 4 & 0 & 0 & 0 & 2 & 0 & 0 & 1 & 3.24 & 24.04 & 395.43 & 395.90 \\
\hline 9 & 0 & 1 & 0 & 0 & 0 & 0 & 0 & 0 & 0 & 1.23 & 29.01 & 438.85 & 440.11 \\
\hline 9 & 0 & 1 & 0 & 0 & 0 & 0 & 0 & 0 & 0 & 1.23 & 29.01 & 438.25 & 440.11 \\
\hline 8 & 1 & 1 & 0 & 0 & 0 & 0 & 1 & 0 & 0 & 1.56 & 28.61 & 437.46 & 436.82 \\
\hline 8 & 0 & 2 & 0 & 0 & 0 & 0 & 1 & 0 & 0 & 1.56 & 28.46 & 435.28 & 435.55 \\
\hline 8 & 2 & 0 & 0 & 0 & 0 & 0 & 0 & 1 & 0 & 1.56 & 28.52 & 434.35 & 436.09 \\
\hline 8 & 2 & 0 & 0 & 0 & 0 & 0 & 0 & 0 & 0 & 1.56 & 28.28 & 433.95 & 434.07 \\
\hline 8 & 0 & 2 & 0 & 0 & 0 & 0 & 0 & 0 & 0 & 1.56 & 27.98 & 433.02 & 431.50 \\
\hline 8 & 1 & 1 & 0 & 0 & 0 & 0 & 0 & 0 & 0 & 1.56 & 28.13 & 431.69 & 432.79 \\
\hline
\end{tabular}




\begin{tabular}{|c|c|c|c|c|c|c|c|c|c|c|c|c|c|c|c|}
\hline 36 & 2,2-dimethyloctane & 8 & 0 & 2 & 0 & 0 & 0 & 1 & 0 & 0 & 0 & 1.56 & 27.65 & 430.05 & 428.68 \\
\hline 37 & 2,4-dimethyloctane & 8 & 0 & 2 & 0 & 0 & 0 & 0 & 0 & 0 & 0 & 1.56 & 27.98 & 429.05 & 431.50 \\
\hline 38 & 3-ethyl-4-methylheptane & 7 & 0 & 1 & 1 & 0 & 0 & 0 & 1 & 0 & 0 & 2.04 & 28.59 & 436.15 & 436.69 \\
\hline 39 & 3,4,5-trimethylheptane & 7 & 2 & 1 & 0 & 0 & 0 & 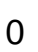 & 2 & 0 & 0 & 2.04 & 28.30 & 435.65 & 434.22 \\
\hline 40 & 3,4,4-trimethylheptane & 7 & 1 & 2 & 0 & U & 0 & U & 2 & 1 & 0 & 2.04 & 28.39 & 434.25 & 434.97 \\
\hline 41 & 5-ethyl-2-methylheptane & 7 & 0 & 1 & 1 & 0 & 0 & 0 & 0 & 0 & 0 & 2.04 & 28.12 & 432.85 & 432.65 \\
\hline 42 & 2,2,3-trimethylheptane & 7 & 1 & 2 & 0 & J & 0 & $\perp$ & 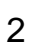 & 0 & 0 & 2.04 & 27.82 & 430.75 & 430.14 \\
\hline 43 & 2,2,6-trimethylheptane & 7 & 0 & 3 & 0 & 0 & 0 & 1 & 0 & 0 & 0 & 2.04 & 26.71 & 422.08 & 420.49 \\
\hline 44 & 2,2,4-trimethylheptane & 7 & 0 & 3 & 0 & 0 & 0 & 1 & 0 & 0 & 0 & 2.04 & 26.71 & 421.45 & 420.49 \\
\hline 45 & 3,3-diethylhexane & 6 & 0 & 0 & 2 & 0 & 0 & 0 & 0 & 1 & 0 & 2.78 & 28.64 & 439.45 & 437.07 \\
\hline 46 & 3,4-diethylhexane & 6 & 0 & 0 & 2 & 0 & 0 & 0 & 1 & 0 & 0 & 2.78 & 28.88 & 437.05 & 439.05 \\
\hline 47 & 4-ethyl-3,3-dimethylhexane & 6 & 2 & 0 & 1 & 0 & 0 & 0 & 2 & 1 & 0 & 2.78 & 28.83 & 436.05 & 438.61 \\
\hline 48 & 3-ethyl-3,4-dimethylhexane & 6 & 2 & 0 & 1 & 0 & 0 & 0 & 2 & 1 & 0 & 2.78 & 28.83 & 435.25 & 438.61 \\
\hline 49 & 2,3,4,4-tetramethylhexane & 6 & 3 & 1 & 0 & 0 & 0 & 0 & 3 & 1 & 0 & 2.78 & 28.38 & 434.75 & 434.89 \\
\hline 50 & 4-ethyl-2,4-dimethylhexane & 6 & 1 & 1 & 1 & 0 & 0 & 0 & 0 & 1 & 0 & 2.78 & 27.72 & 434.25 & 429.24 \\
\hline 51 & 4-ethyl-2,3-dimethylhexane & 6 & 1 & 1 & 1 & 0 & 0 & 0 & 2 & 0 & 0 & 2.78 & 28.43 & 434.05 & 435.34 \\
\hline 52 & 2,2,3,3-tetramethylhexane & 6 & 2 & 2 & 0 & 0 & 0 & 1 & 4 & 1 & 0 & 2.78 & 28.38 & 433.46 & 434.88 \\
\hline 53 & 2,2,3,4-tetramethylhexane & 6 & 2 & 2 & 0 & 0 & 0 & 1 & 3 & 0 & 0 & 2.78 & 27.66 & 431.95 & 428.76 \\
\hline 54 & 2,3,4,5-tetramethylhexane & 6 & 2 & 2 & 0 & 0 & 0 & 0 & 3 & 0 & 0 & 2.78 & 27.99 & 429.35 & 431.57 \\
\hline 55 & 2,3,3,5-tetramethylhexane & 6 & 2 & 2 & 0 & 0 & 0 & 0 & 2 & 1 & 0 & 2.78 & 27.75 & 426.25 & 429.54 \\
\hline 56 & 3-ethyl-2,2,3-trimethylpentane & 5 & 1 & 2 & 1 & 0 & 0 & 1 & 4 & 1 & 0 & 4.00 & 28.79 & 442.65 & 438.31 \\
\hline 57 & 2,2,3,3,4-pentamethylpentane & 5 & 2 & 3 & 0 & 0 & 0 & 1 & 6 & 1 & 0 & 4.00 & 28.82 & 439.20 & 438.60 \\
\hline 58 & undecane & 11 & 0 & 0 & 0 & 0 & 0 & 0 & 0 & 0 & 0 & 1.00 & 32.87 & 469.08 & 470.40 \\
\hline 59 & 2,2-dimethylnonane & 9 & 0 & 2 & 0 & 0 & 0 & 1 & 0 & 0 & 0 & 1.49 & 30.39 & 450.19 & 451.33 \\
\hline 60 & 4,5-dipropyloctane & 8 & 0 & 0 & 0 & 0 & 2 & 0 & 1 & 0 & 0 & 3.06 & 36.21 & 493.00 & 494.36 \\
\hline 61 & 3-methyltetradecane & 14 & 1 & 0 & 0 & 0 & 0 & 0 & 0 & 0 & 0 & 1.15 & 43.00 & 538.19 & 537.88 \\
\hline 62 & 2,3-dimethyltridecane & 13 & 1 & 1 & 0 & 0 & 0 & 0 & 1 & 0 & 0 & 1.33 & 42.37 & 536.79 & 534.09 \\
\hline 63 & 6-propyldodecane & 12 & 0 & 0 & 0 & 0 & 1 & 0 & 0 & 0 & 0 & 1.56 & 40.97 & 524.75 & 525.53 \\
\hline 64 & 2-methylpentadecane & 15 & 0 & 1 & 0 & 0 & 0 & 0 & 0 & 0 & 0 & 1.14 & 45.62 & 553.99 & 553.12 \\
\hline 65 & 2,3-dimethylpentadecane & 15 & 1 & 1 & 0 & 0 & 0 & 0 & 1 & 0 & 0 & 1.28 & 47.90 & 568.79 & 565.78 \\
\hline 66 & eicosane & 20 & 0 & 0 & 0 & 0 & 0 & 0 & 0 & 0 & 0 & 1.00 & 57.87 & 616.95 & 615.73 \\
\hline 67 & 3-methylnonadecane & 19 & 1 & 0 & 0 & 0 & 0 & 0 & 0 & 0 & 0 & 1.11 & 56.87 & 609.19 & 611.07 \\
\hline 68 & 2,3-dimethyloctadecane & 18 & 1 & 1 & 0 & 0 & 0 & 0 & 1 & 0 & 0 & 1.23 & 56.21 & 611.99 & 607.94 \\
\hline 69 & docosane & 22 & 0 & 0 & 0 & 0 & 0 & 0 & 0 & 0 & 0 & 1.00 & 63.43 & 641.75 & 640.38 \\
\hline 70 & tricosane & 23 & 0 & 0 & 0 & 0 & 0 & 0 & 0 & 0 & 0 & 1.00 & 66.21 & 653.25 & 652.00 \\
\hline 71 & octacosane & 28 & 0 & 0 & 0 & 0 & 0 & 0 & 0 & 0 & 0 & 1.00 & 80.10 & 704.75 & 704.24 \\
\hline 72 & triacontane & 30 & 0 & 0 & 0 & 0 & 0 & 0 & 0 & 0 & 0 & 1.00 & 85.66 & 722.75 & 722.85 \\
\hline
\end{tabular}

